\title{
Organ-on-a-Chip Platforms for Drug Delivery and Cell Characterization: A Review
}

\author{
Zongjie Wang ${ }^{\dagger}$, Roya Samanipour ${ }^{\dagger}, \mathrm{Kyo}-\mathrm{in} \mathrm{Koo}^{1, *}$ and Keekyoung Kim ${ }^{* *}$ \\ School of Engineering, The University of British Columbia, Kelowna, BC, V1V 1V7, Canada \\ ${ }^{1}$ Department of Biomedical Engineering, University of Ulsan, Ulsan 680-749, Republic of Korea
}

(Received December 6, 2014; accepted April 27, 2015)

Key words: organ-on-a-chip, microfluidics system, drug screening and delivery, cellular microenvironment, tissue engineering

Developments in micro- and nanofluidic technologies have led to new kinds of cell culture and screening systems that are collectively termed organ-on-a-chip systems. Organ-on-a-chip systems are in vitro microfabricated devices that mimic dynamic interactions of in vivo microenvironments. In addition to existing two-dimensional and three-dimensional tissues, organ-on-a-chip systems can mimic the biomechanical and biochemical microenvironments of in vivo tissues as well as the interactional effects of the microenvironments on cell and tissue functions. Owing to those features, organ-ona-chip systems have become excellent platforms for drug screening and delivery tests. In this review, specific examples of organ-on-a-chip devices and their applications in tissue engineering and drug delivery tests are presented. The utility and performance of stateof-the-art organ-on-a-chip systems, including lung-on-a-chip, heart-on-a-chip, vessel-ona-chip, liver-on-a-chip, and tumor-on-a-chip, are also covered in this review. Limitations of conventional systems, basic fabrication processes for organ-on-a-chip devices, and future prospects of organ-on-a-chip systems are discussed.

\section{Introduction}

How in vivo microenvironments are constructed and how cells interact with other cells and the surrounding environments have been unresolved. Knowledge of these interactions will significantly improve our understanding of the nature of the human body at the tissue and cellular levels. ${ }^{(1)}$ In addition, the development of new drugs and disease therapies will benefit if the interaction between specific cells and drugs can be screened directly. ${ }^{(2)}$ In the longer term, the goal is to directly and precisely mimic the in vivo microenvironments and to understand their internal interactions with drugs. Many direct and indirect methods have been developed. ${ }^{(3-6)}$

\footnotetext{
${ }^{*}$ Corresponding author: e-mail: kikoo@ulsan.ac.kr

${ }^{* *}$ Corresponding author: e-mail: keekyoung.kim@ubc.ca

$\dagger$ These authors contributed equally to this work.
} 
The mainstream indirect method is the animal test. The effects of the inputs (e.g., drugs) are evident by outputs, such as the concentration changes of specific chemicals in animals' body fluids. (7) However, during animal testing, it is difficult to determine the internal processes by measuring the inputs and outputs. Also, in vivo microenvironments of animals are slightly different from those of human beings, which decreases the reliability of the results of animal tests. ${ }^{(8)}$ More seriously, animal tests often cause ethical problems and discarding the animals used in the tests consumes both time and resources. ${ }^{(8)}$ To avoid these shortcomings, people have tried to culture human cells and screen drugs in vitro. However, the characteristics of cells cultured in vitro are significantly different from those of cells cultured in vivo owing to failure to reconstruct nearby cellular microenvironments. ${ }^{(9)}$ The deficiencies of such methods have enormously increased the time and money for drug discovery. Although many drugs are discovered every year, few can be commercialized because their potential effects on the human body remain unclear. ${ }^{(8)}$

Fortunately, with the development of nanotechnology, scientists can precisely manipulate cellular microenvironments at the micro- and nanoscales. In addition, using microfabrication techniques, such as soft lithography, highly accurate devices can be developed at reasonable costs with a marked reduction of the use of chemicals and biological materials. ${ }^{(10,11)}$ However, rebuilding the cellular microenvironment of particular tissues in vitro in a simple and straightforward manner remains challenging. To overcome the challenge, organ-on-a-chip systems have been developed; these represent a combination of microfluidic and cell biological techniques.

Organ-on-a-chip devices aim to duplicate the function and microstructure of multicellular human organs in vitro on a microfluidics chip. ${ }^{(1,12)}$ Such devices include the biological functions of organs and the biochemical, bioelectrical, and biomechanical properties of cellular microenvironments and extracellular matrixes (ECMs). Recent studies have demonstrated the feasibility of organ-on-a-chip systems, which can closely mimic in vivo tissues and provide platforms for drug delivery test and biological cell characterization..$^{(1,8,13)}$

In this review, specific examples of different types of organ-on-a-chip devices, such as lung-on-a-chip, heart-on-a-chip, vessel-on-a-chip, liver-on-a-chip, and tumor-on-achip, are presented. Working principles of those devices as well as their applications are provided. Moreover, the limitations of the current systems, basic fabrication methods for microfluidic devices, and future prospects of organ-on-a-chip are introduced.

\section{Limitations of Conventional Systems}

In this section, the limitations of conventional drug screening and delivery test systems are reviewed. A typical drug development flow contains pre-clinical, clinical and post-marketing phases. ${ }^{(14)}$ In vitro drug screening (bench test) and drug delivery test (currently, mostly animal test) are the two most important pre-clinical phase steps. ${ }^{(14,15)}$

To monitor the cell behavior of drugs in vitro, platforms mimicking in vivo environments in the human beings have emerged. In vitro models can support almost all types of drug screening methods including free drugs, drug-delivery agents, implanted 
depots, liposomes, and nanoparticles. ${ }^{(16)}$ At the early stage, most in vitro platforms are based on two-dimensional (2D) cell culturing platforms, ${ }^{(17,18)}$ as shown in Fig. 1(a). The platforms first seed a monolayer of cells on the culturing flask. Then, drugs for screening are added into the cell culture media. After certain times, the cell responses to the drugs are recorded. Finally, the chemical and biological signals produced by cells are analyzed. Those $2 \mathrm{D}$ in vitro platforms provide a direct procedure to screen interactions between drugs and cells. However, many studies have shown that a 2D cell monolayer cannot adequately mimic three-dimensional (3D) cellular microenvironments in the human body, which results in the oversimplification of the ECM. ECM properties of in vitro and in vivo structures are remarkably different. ${ }^{(9)}$ Thus, how the drugs act in the in vivo microenvironment is likely difficult to fathom using solely $2 \mathrm{D}$ in vitro tests.

Many 3D culturing platforms have been developed in response to this limitation. ${ }^{(19-22)}$ The most common 3D cell culturing platforms are cell aggregates, ${ }^{(21)}$ spheroids, ${ }^{(19)}$ and cell sheets. ${ }^{(18,22)}$ Examples of cell aggregates and multicell-type sheets are shown in Figs. 1(b) and 1(c), respectively. Figure 1(d) shows microscopy images of the fabricated 3D cell structures. Cells accumulate to form specific 3D structures, such as aggregates or multicellular layers. In such structures, cells contact each other in three dimensions. These platforms successfully create the 3D ECM microenvironments in vitro. However,

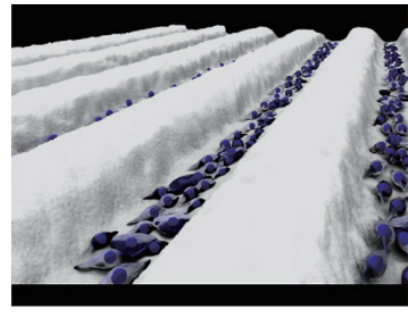

(a)

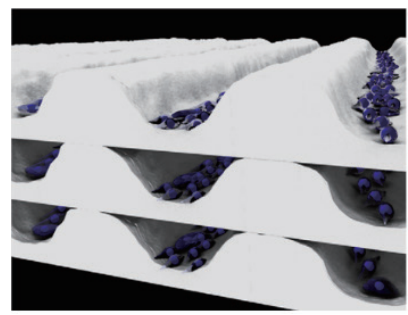

(c)

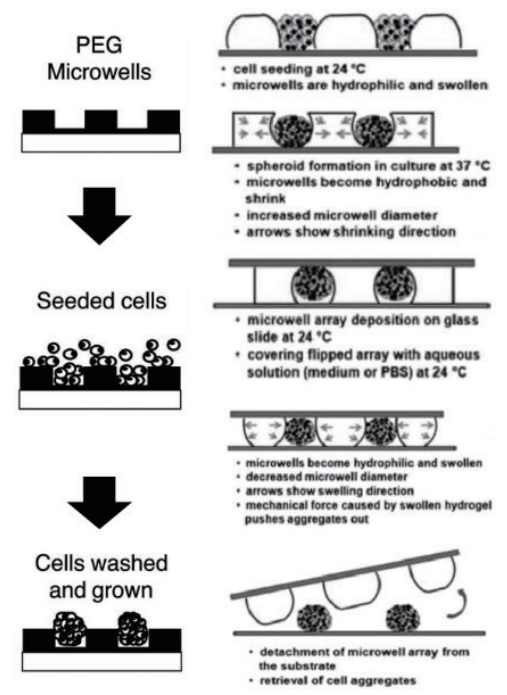

(b)
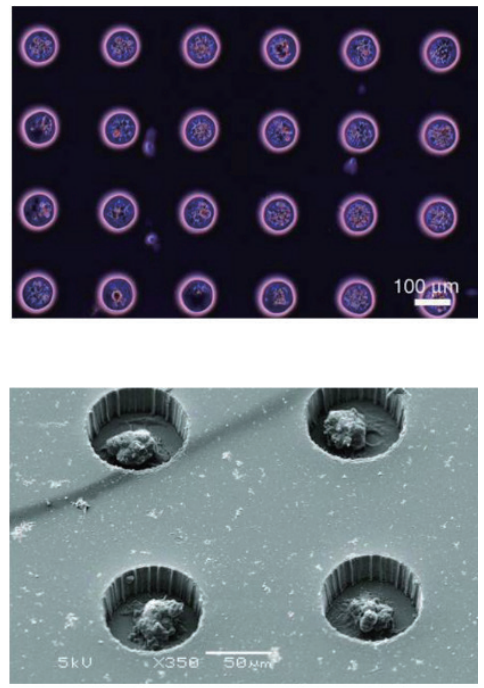

(d)

Fig. 1. (Color online) 2D and 3D cell culturing platforms. (a) Schematic of 2D flat cell culturing platform. ${ }^{(18)}$ (b) Schematic of 3D cell culturing platform. Upper: microscale wells to induce cell aggregation. ${ }^{(86)}$ Lower: mold-based cell aggregates. ${ }^{\left({ }^{87}\right)}$ (c) Multilayer 3D cell sheets with different types of cells. ${ }^{(18)}$ (d) Light microscopy and scanning electron microscopy images of cell aggregates in microwells. ${ }^{(86)}$ 
the interaction between the cellular microenvironments does not only include chemical responses but also mechanical and electrical interactions that can significantly affect cell behaviors. ${ }^{(23)}$ One explicit example is the lungs. During inhalation, the volume of the lung changes markedly, subjecting the lung cells to non-negligible mechanical stress. ${ }^{(24)}$ Also, changes in volume play an important role in increasing the surface-area-to-volume ratio, which is a critical parameter for air exchange. To study in vivo cell responses to drugs, we must consider mechanical and electrical changes in cells, as well as chemical changes. Hence, similarly to $2 \mathrm{D}$ platforms, passing $3 \mathrm{D}$ in vitro screening tests cannot guarantee that drugs are safe for humans owing to the absence of data on mechanical and other essential cell effects. ${ }^{(25,26)}$

A drug delivery test determines the metabolism and toxicological effects of drugs. ${ }^{(7)}$ The drug delivery test is considered as the most crucial step before clinical trials because it can predict the performance of a new drug inside the human body.(27) Traditional drug delivery tests are based on animal models. The in vivo microenvironment in animals is the closest to humans in nature. However, animal models have many limitations as a preclinical test platform for drugs. It is difficult to achieve reproducible test results of drug delivery using animal models. ${ }^{(8)}$ The characteristics of each animal are not controllable and animal-to-animal variation results in different test outcomes that can appreciably reduce the reliability of animal models. Additionally, the metabolism of drugs is hard to deduce by animal tests, since animal models only guarantee case-by-case results. ${ }^{(28)}$ In most cases, researchers can only investigate the effect of drugs by monitoring the changes in the concentrations of chemical compounds. ${ }^{(29)}$ Such an indirect monitoring method inhibits the deeper understanding of toxicity and mechanisms of interactions as well as the long-term stability of drugs. Although the magnetic resonance imaging (MRI) of the whole body is one of the possible solutions for tracing the drug delivery process, high cost and limited resolution markedly reduce its potential usage for drug delivery test. ${ }^{(7)}$

To investigate the mechanical and other interactions in the cellular microenvironment, the organ-on-a-chip platform has been developed. On the basis of the advancements of polymer material science and nanofabrication technology, such platforms can facilitate the study of cell-cell, ${ }^{(24)}$ cell-ECM ${ }^{(30)}$ and cell-tubular flow ${ }^{(31)}$ interactions. The platforms allow scientists to carry out drug screening and delivery tests and analyses. In addition, organ-on-a-chip systems can be employed to characterize cell physical properties in the microenvironment. ${ }^{(32)}$ The advantages of organ-on-a-chip systems include low cost, high controllability, and the ability to mimic organ functions dynamically. ${ }^{(1,8,12,33)}$

\section{Principles of Microfabrication}

To precisely mimic in vivo microenvironments, organ-on-a-chip platforms rely heavily on the fabrication processes. Microfabrication techniques have been widely used to fabricate organ-on-a-chip platforms because of the possibility of building tissue environments at the microscale. ${ }^{(34)}$ As shown in Fig. 2, a variety of techniques including replica molding, ${ }^{(35)}$ soft lithography, ${ }^{(35)}$ and microcontact printing ${ }^{(36)}$ have been utilized to fabricate organ-on-a-chip platforms. 


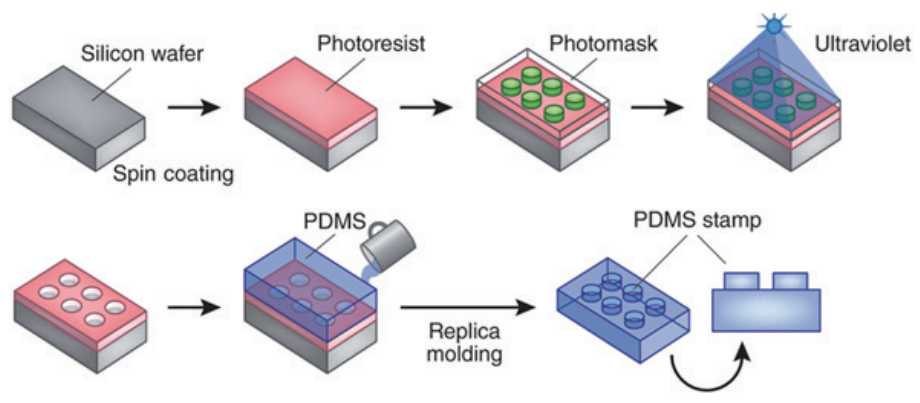

(a)

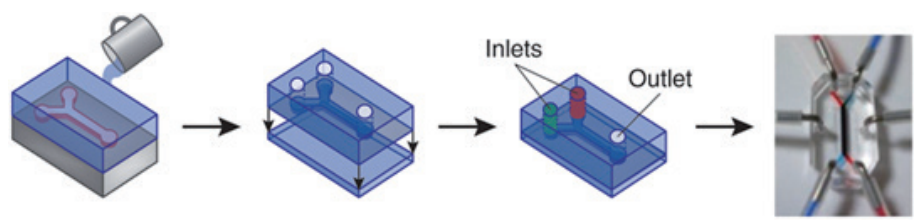

(b)

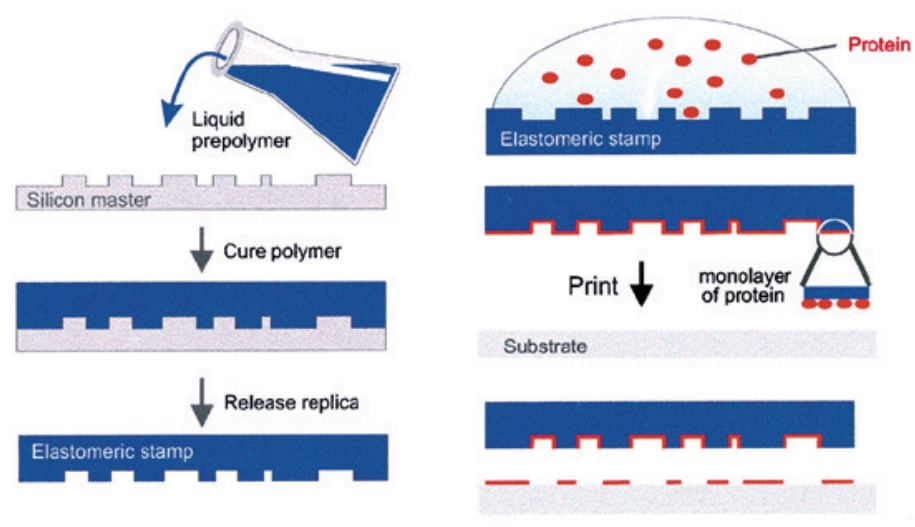

(c)

Fig. 2. (Color online) Microfabrication techniques and applications. (a) Use of replica molding to create PDMS stamps. (b) Use of soft lithography to generate a single PDMS channel with two inlets and one outlet. ${ }^{(88)}$ (c) Microcontact-printed proteins used to pattern cells. ${ }^{(36)}$

The most widely used fabrication material is polydimethylsiloxane (PDMS), reflecting its biocompatibility and flexibility. ${ }^{(10)}$ PDMS is optically clear, which allows the capture of high-quality images of cultured cells. The PDMS stamp shown in Fig. 2(a) was fabricated using the replica molding technique. ${ }^{(35)}$ In this design, the silicon wafer is coated with a uniform photoresist layer. The photoresist can either be crosslinked or uncrosslinked by exposure to UV light. The use of a photomask permits only the desired parts of the photoresist to be illuminated. After this step, the pattern is 
transferred to the silicon wafer substrate, which becomes a mold. The PDMS is poured on the patterned mold and is polymerized during baking in an oven. The PDMS device is then peeled off from the substrate. The substrate can be reused for many fabrications; hence, the process is termed replica molding. Replica molding is a subtechnology of soft lithography. Soft lithography can also be used to fabricate PDMS channels for flowing fluids [Fig. 2(b)]. The fabrication steps are similar to the replica molding method, with the sole difference being that the PDMS device is further bonded with other substrates (usually glass slides or other complementary PDMS layers). The liquid flow in the microfluidic channels is a laminar flow in a microscale. Laminar flow can be controlled to study cell-flow interaction. Hence, the use of a microfluidic chip with controllable laminar flows allows the study of cell aggregation, ${ }^{(37)}$ cardiac tissue formation, ${ }^{(38)}$ as well as differentiation. ${ }^{(39)}$ Microfabrication techniques also allow the fabrication of porous membranes to mimic the vascular endothelium and parenchymal tissues by culturing two different kinds of cells on both sides of the porous PDMS substrate. ${ }^{(24)}$ The pattern of cultured cells can be controlled by microcontact printing technology, ${ }^{(36)}$ as shown in Fig. 2(c). After fabricating a PDMS membrane, an elastomeric PDMS stamp is generated and coated with a monolayer of proteins. By stamping the PDMS stamp on the substrate, the desired areas of the proteins can be transferred to the membrane substrate. Since cells only grow in the area containing proteins, by controlling the pattern of the printed proteins, the cells on the membrane can be patterned as well.

Microwells, fluidic channels, and porous membranes are the most widely used components for organ-on-a-chip platforms constructed to study in vivo interactions, such as cell-cell interactions, cell behaviors, and cell responses to chemical and mechanical stimulations. ${ }^{(35)}$ Microfabrication technology can be used to build and assemble the components of organ-on-a-chip platforms easily, making it possible for the design of in vitro systems to investigate in vivo microenvironmental effects.

\section{Existing Organ-on-a-Chip Platforms}

In this section, various existing state-of-the-art organ-on-a-chip platforms are introduced to show the feasibility and potential of the system for biomedical applications. In the six subsections, which include vessel-on-a-chip, liver-on-a-chip, heart-on-achip, lung-on-a-chip, and tumor-on-a-chip, working principles and device structures are discussed.

\subsection{Vessel-on-a-chip}

Blood vessels are responsible for transporting blood through the body. Blood is carried away from the heart by arteries. Nanoparticles in blood vessels diffuse through the capillary endothelial barrier to exchange water and chemicals between tissues and blood. Finally, blood is carried back from capillaries to the heart by veins. It is important to study the interaction of these carriers with the vasculature and also the effects of fluid shear stress and cyclic stretch on endothelial cells. In some studies, the geometric characteristics of the vasculature, which includes simple channels, ${ }^{(40-42)}$ complex parameters, ${ }^{(43)}$ and the accumulation of micro- and nanoparticles inside the 
microchannels, have been investigated. ${ }^{(44)}$

The other important parameters to be considered are the fluid shear stress and cyclic stretch on endothelial cells. ${ }^{(45-48)}$ The vessel-on-a-chip is the microfluidic device fabricated to mimic the blood vessels. In most studies, two parallel-flow PDMS chambers separated from a membrane are fabricated to culture vascular endothelial cells on the membrane. Microfluidic chambers are connected to syringe pumps that control flow rates [Figs. 3(a) and 3(b)]. The shear stresses generated by the flow rate and applied to endothelial cells can be studied. ${ }^{(49)}$

Vascular endothelial cells under cyclic stretching are one of the important factors to study. ${ }^{(50)}$ An upper microfluidic channel layer and a bottom groove layer are separated by an elastic membrane that applies cyclic stretching strain to the cells. The groove

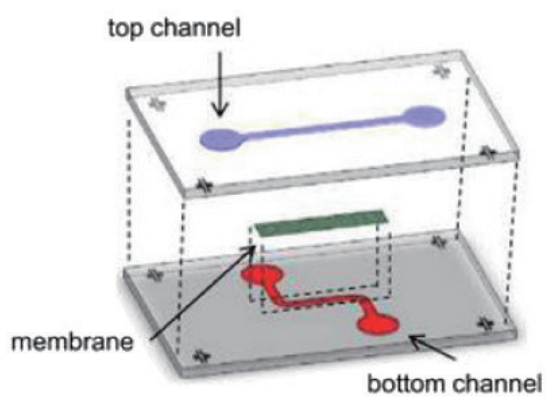

(a)

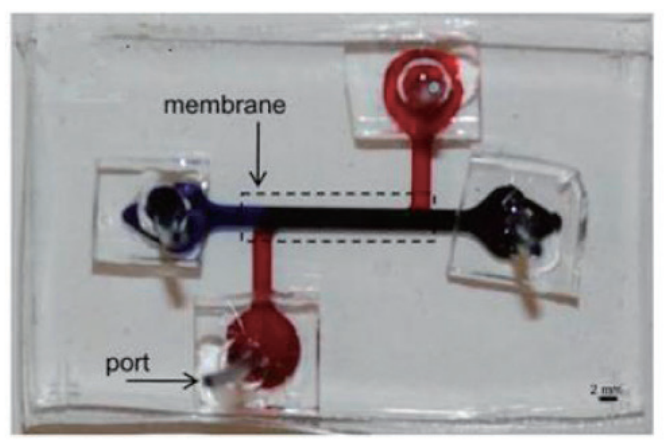

(b)

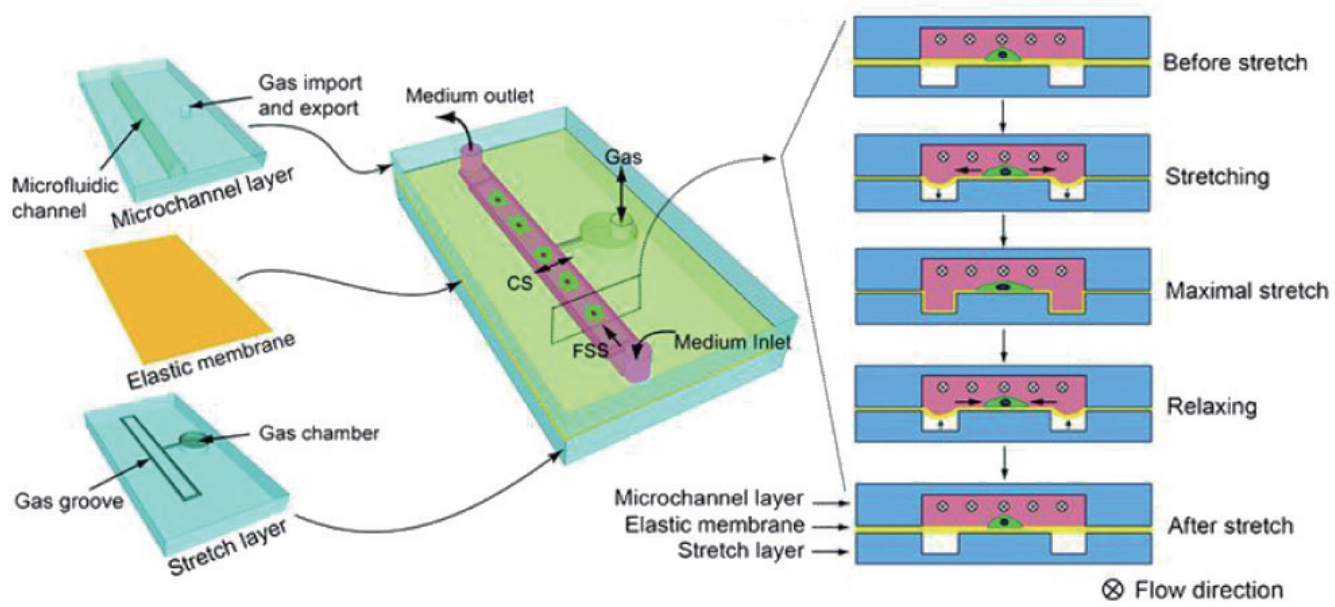

(c)

(d)

Fig. 3. (Color online) Vessel-on-a-chip. (a) Schematic of two PDMS chambers connected by a membrane.(49) (b) Realization of the devices shown in (a). (c) Long microfluidic channel with consecutive flow. (d) Repeatedly stretched and relaxed elastic membrane. ${ }^{(50)}$ 
layer is attached under the elastic membrane and is connected to a vacuum pump that applies suction pressure to stretch the elastic membrane. The cells are cultured on top of the membrane and fluid flow is passed through the upper channel layer while vacuum pressure is applied to the stretched cells on the elastic membrane. The cells cultured on the membrane are cyclically stretched and relaxed [Figs. 3(c) and 3(d)].(50)

\subsection{Liver-on-a-chip}

The liver is a vital organ in the body that plays a primary role in the digestive system in detoxification, protein synthesis, and blood filtering. ${ }^{(51)}$ Liver-on-a-chip platforms have been developed to understand the liver function better and to study drug hepatotoxicity and metabolism. ${ }^{(31,52,53)}$

A microfluidic device fabricated to mimic the permeable sinusoidal endothelial barrier between hepatocytes and the liver sinusoid ${ }^{(31)}$ includes three main sectionsa central channel to contain the hepatocytes, a microfluidic sinusoid barrier patterned with a set of narrow ( $2 \mathrm{~mm}$ wide) microchannels to model the endothelial barrier, and a microfluidic convection channel surrounding the barrier [Fig. 4(a)]. The flow of nutrients through the convection microfluidic channel feeds the hepatocytes. This system mimics the transportation between blood flow and hepatocytes, and the shear stress over hepatocytes with a patterned set of microchannels.

3D microengineered hepatic cell culturing platforms have been introduced to remodel the complex microenvironment of the liver. ${ }^{(54-56)}$ The use of a perfused multiwell plate enabled the study of drug toxicity and metabolism [Fig. 4(b)]. ${ }^{(53)}$ This device consists of a perfused multiwell with an array of 12 bioreactors. The bioreactors including hepatocytes, liver sinusoidal endothelial cells, and stellate and Kupffer cells are placed in scaffold wells. The scaffold wells are continuously perfused by means of micropumps. Cell-cell signaling (paracrine signaling) of hepatocytes and hepatic stellate cells (HSCs) have been investigated. ${ }^{(52)}$ In that study, the fabricated 3D microfluidic liver-on-a-chip consisted of two different chambers. In one chamber, HSCs were cultured on a flat chamber. In the other chamber, hepatocytes were cultured on a concave chamber to avoid direct cell-cell contact. These two different cells were connected with a tube and one osmotic pump, and adjusted to control the flow rate of a nutrient solution flow [Fig. $4(c)]$.

\subsection{Heart-on-a-chip}

The development of heart-on-a-chip platforms is one of the most challenging areas in organ-on-a-chip research. The challenges are to mimic dynamic cardiac tissue microenvironments. It is difficult to simultaneously study the contractility and electrophysiological responses of cardiac tissues. Nevertheless, a few studies have been carried out with cardiac tissue engineering to mimic cardiac tissue microenvironments ${ }^{(57)}$ and with heart-on-a-chip platforms to study the contractility and electrophysiological response of cardiac tissue simultaneously (Fig. 5). ${ }^{(58)}$

At the early stage of the development of the heart-on-a-chip, a PDMS microfluidic network was combined with a planar electrode array to measure the extracellular potential from individual adult cardiomyocytes. ${ }^{(59)}$ Another microfluidic device with 


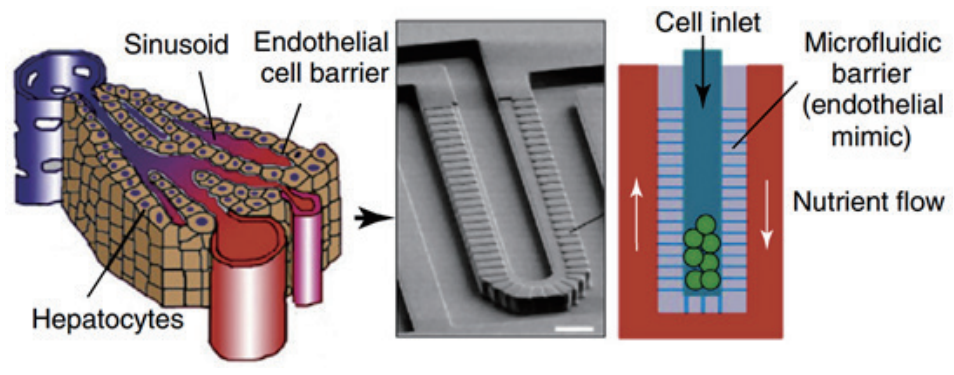

(a)

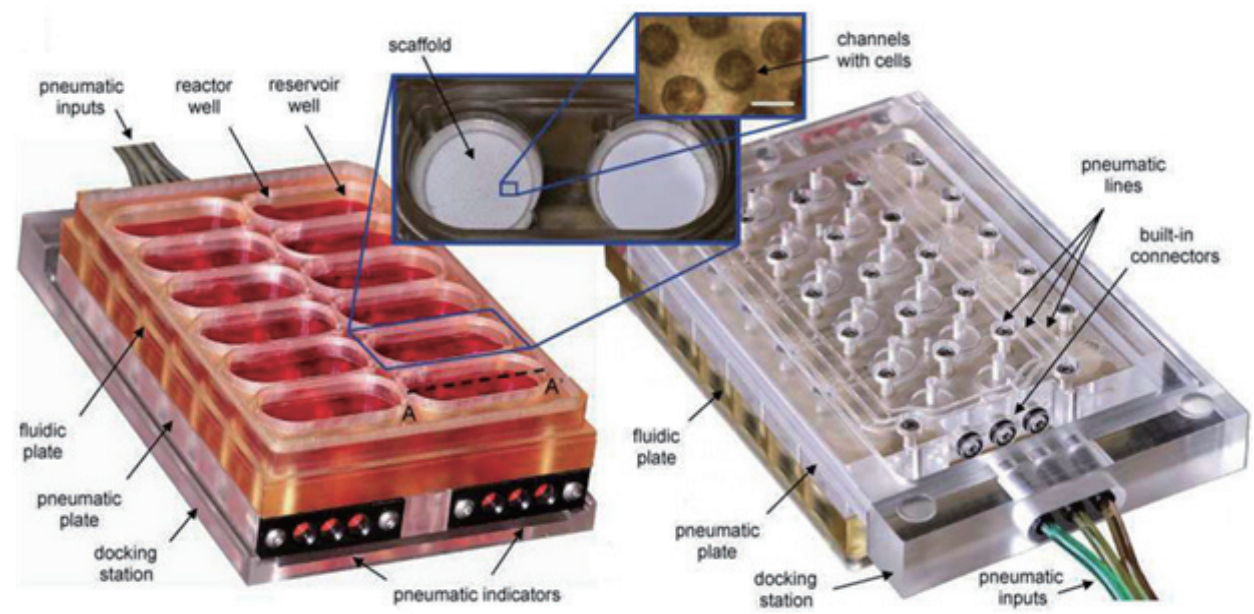

(b)

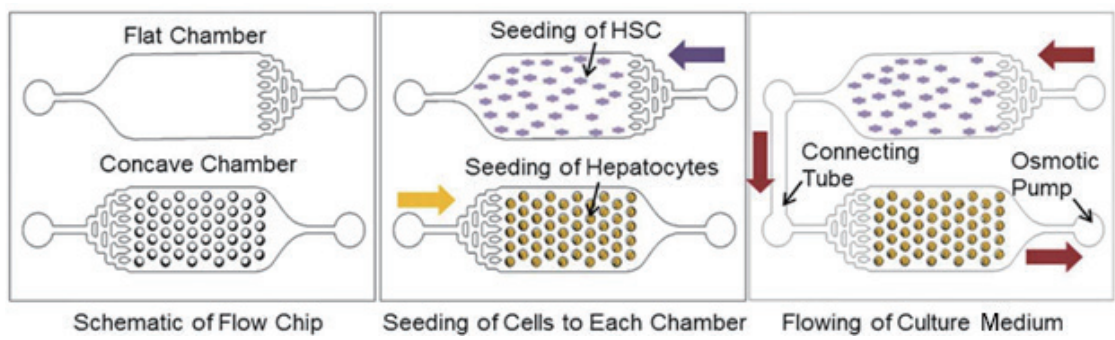

(c)

Fig. 4. (Color online) Liver-on-a-chip. (a) Microfluidic liver on a chip consisting of a central liver cell chamber and surrounding nutrient flow chamber to mimic the structure of the liver. ${ }^{(31)}$ (b) Microengineered perfusion of the hepatic culture. ${ }^{(53)}$ (c) 3D microfluidic liver on a chip consisting of two different chambers connected with a tube. ${ }^{(52)}$ 


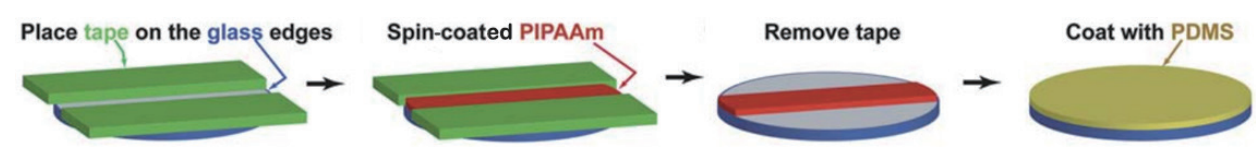

(a)

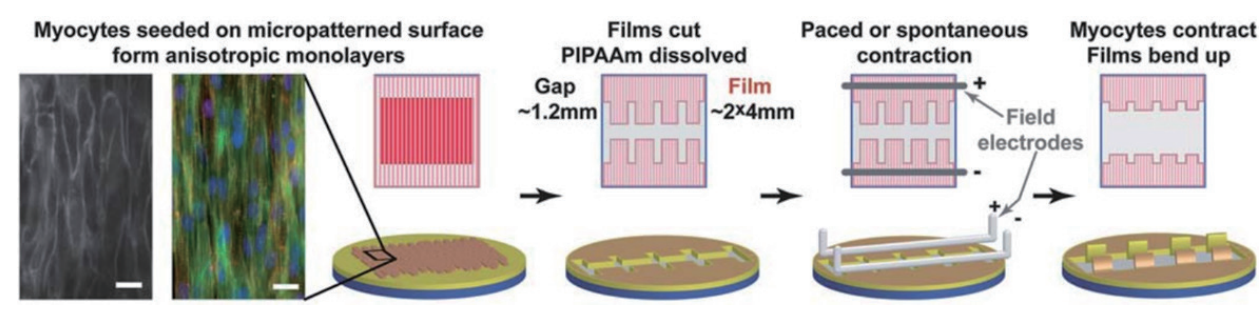

(b)
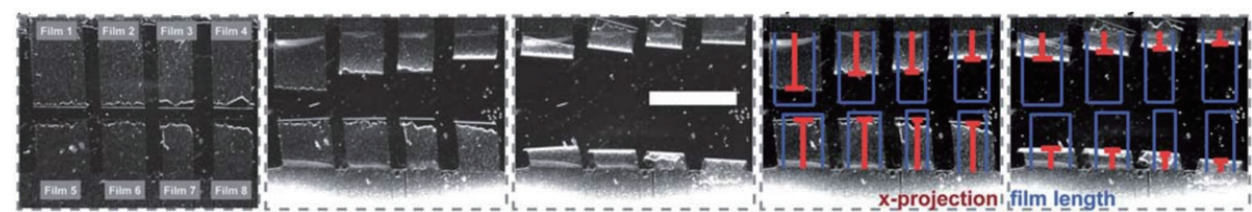

(c)

Fig. 5. (Color online) Heart-on-a-chip devices and the image of their beating. ${ }^{(58)}$

an array of electrodes was developed to electrically measure the metabolic profile of cardiomyocytes and optically measure cell contractility. ${ }^{(60)}$ Grosberg et al. first introduced a tissue-level heart-on-a-chip to measure the contractility of neonatal cardiac muscle tissue. ${ }^{(58)}$ In the design, eight muscular thin films (MTFs) were fabricated on a chip. A layer of poly(N-isopropylacrylamide) (PIPAAm) dissolved at a temperature below $35{ }^{\circ} \mathrm{C}$ was spin-coated on top of a glass slide [Fig. 5(a)]. Subsequently, a PDMS layer was coated on top of the PIPAAM layer. The PDMS layer was used to seed neonatal rat ventricular cardiomyocytes. The substrate seeded with cells was placed in a bath and the film layers were manually cut to fabricate an array of two opposite rows of four rectangular MTF layers. The MTFs were peeled off after PIPAAm was dissolved when the solution was kept below $35{ }^{\circ} \mathrm{C}$. Finally, electrodes were placed on top and at the bottom of the MTFs. The MTFs started to curl up when electrical stimulation from the field electrode was carried out [Fig. 5(b)]. Finally, the deflection of cells responding to electrical stimuli was measured [Fig. 5(c)].

\subsection{Lung-on-a-chip}

The lung is another vital organ where external oxygen gas is exchanged with carbon dioxide in the blood. Investigating cell-cell interactions, cell-blood flow, and cell-gas flow in the respiratory tract is essential for both physiological research and drug delivery 
tests. Some microfluidic platforms are designed to culture lung cancer cells and screen their response to drugs ${ }^{(61)}$ and electrical stimulation. ${ }^{(62)}$ However, these platforms do not mimic both the deformation of the lung tissue during breathing and the bi-cell barriers between blood and air. Thus, this type of microfluidic system is limited to cell-level characterizations.

Huh et al. presented the first lung-on-a-chip system. ${ }^{(24)}$ The structure of the lungon-a-chip system is shown in Fig. 6. This device consists of three channels embedded: two side channels and one main channel divided by a membrane. The membrane and side channels are the main components of this system. After seeding endothelial and epithelial cells on each side of the porous membrane, the main channel can mimic the alveolar-capillary barrier in the lungs, allowing the interaction between two types of cells and two flows in the main channel. For example, immune cells from blood flow can cross through microholes in the membrane barrier and kill Escherichia coli in the airway flow. The suction pressure on each side of the chamber can be controlled to adjust the membrane stretch in the main channel, which mimics the lung breathing movement. Great improvements have been made to verify the in vitro microfluidic lung-

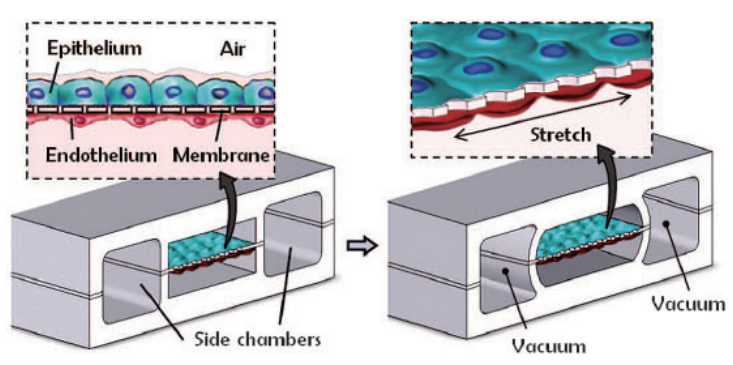

(a)

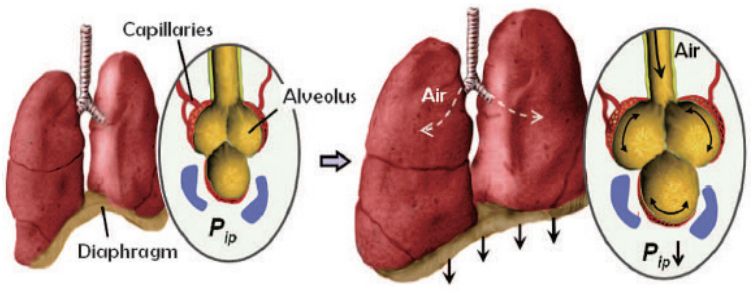

(b)
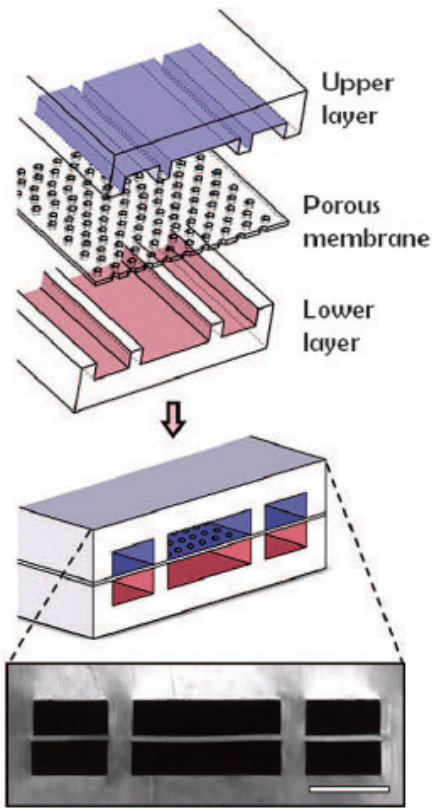

(c)

Fig. 6. (Color online) Typical lung-on-a-chip system.(24) (a) PDMS-based membrane mimicking the alveolar-capillary barrier and a vacuum-based deformation controller mimicking the breathing movements of the lung. (b) Schematic representations of lung size changing during the inhalation. (c) Bonding and alignment of three-layer PDMS structures to form the devices. 
on-a-chip platform. Compared with traditional platforms, the lung-on-a-chip allows the investigation of complex chemical, mechanical, and biological effects, providing a tangible platform for characterizing dynamic cell behaviors in vitro as well as the comprehensive tests of drug effects.

Other types of lung-on-a-chip systems rely on the static characteristics of lung cells to realize specific functions. ${ }^{(63-66)}$ Most of the static lung-on-a-chip platforms aim to detect lung cancer cell markers or the response of screen lung tumor cells to microenvironmental changes. ${ }^{(63-65)}$ The device geometry and flow rate can be optimized to better mimic in vivo lung functions by applying the finite element method (FEM) and computational fluid dynamics (CFD). ${ }^{(66)}$

\subsection{Tumor-on-a-chip}

The tumor-on-a-chip is one of the major areas of interest in organ-on-a-chip research. The emigration and vascularization mechanisms of tumor cells still remain unclear. Although nanoparticles can be used to deliver drugs to targeted cancer cells, the process and side effects of the nanoparticles are difficult to analyze. Existing tumor-on-a-chip platforms can be divided into three subcategories. The first subcategory monitors the emigration and vascularization of tumor cells, especially antiangiogenesis. ${ }^{(67)}$ The second sub-category aims to screen the effect of nanoparticles encapsulating drugs on cancer cells. ${ }^{(30)}$ The third subcategory focuses on detecting cancer markers from blood samples for early stage cancer detection. ${ }^{(65,68-70)}$

In the beginning, microfluidic devices were used to generate 3D tumor spheroids with controllable density and diameter. ${ }^{(72,73)}$ Microfluidic devices have also been used to sort different cancer cells ${ }^{(73)}$ and balance cell density for multi-inflow. ${ }^{(74)}$ To investigate the interaction between normal cells and tumors, several 3D platforms have been developed to co-culture normal and tumor cells. ${ }^{(64)}$ Although 3D platforms cannot be considered as organ-on-a-chip devices, they enable further investigation of in vitro tumor cell behavior.

The most common tumor-on-a-chip platforms are cancer marker detection devices. Usually, microfluidic devices with specially design channel structures can collect circulating tumor cells. ${ }^{(65,68-70)}$ Nanoparticle screening platforms to create a microenvironment that mimics tumor tissues and nearby vessels are also widely used in drug screening and delivery systems. ${ }^{(30)}$ A tumor-on-a-chip device mimicking breast cancer is schematically diagrammed in Fig. 7. A group of solid tumor cells and a pair of capillary and lymphatic vessels are mimicked in the 3D structure. The top channel (red channel) mimics the capillary with endothelium using a monolayer of endothelial cells on a porous membrane. The bottom layer has a center channel mimicking breast tumor cells as well as two side channels mimicking the lymphatic system. Using this platform, the transport of nanoparticles and the dynamic interaction between cancer cells and nanoparticles can be screened. During experiments, nanoparticles were transported from the top channel to the tumor channel through the porous membrane. After the nanoparticles interact with tumor cells, the byproducts were observed in the side lymphatic channel. Parameters including membrane pore size and fluid pressure affected the efficiency of nanoparticles for delivering drugs, providing valuable guidance for the design of nanoparticles. In addition to screening the interaction between tumor cells and 


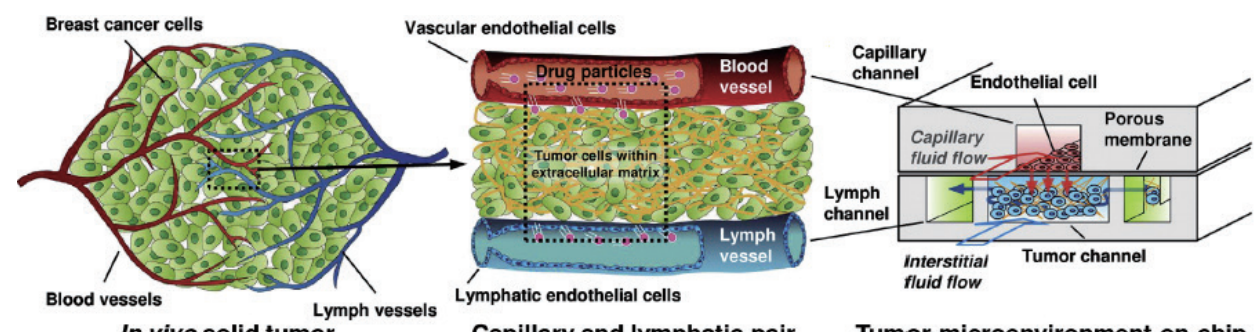

(a)

(b)
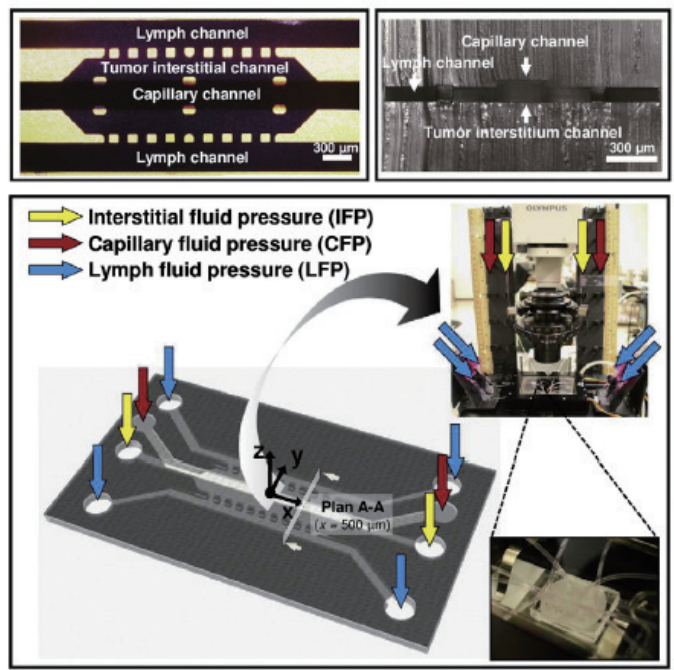

(c)

Fig. 7. (Color online) Tumor-on-a-chip devices. ${ }^{(30)}$ (a) In vivo breast tumor microenvironment formed vascular endothelial, lymphatic endothelial, and tumor cells. (b) Conceptual design: schematic of the tumor-microenvironment-on-chip. (c) Fabricated prototype and schematic of the perfusion setup of the tumor-on-a-chip device.

nanoparticles, the transportation of nanoparticles in vessel tissues has been investigated to help create better nanoparticles. ${ }^{(75)}$

Platforms for screening vascularization are still in the early stage of development. No long-term study of the formation of blood vessels has been carried out in microfluidic channels. To investigate the short-term relationship between cancer cells and blood vessels, the microfluidic platform shown in Fig. 8 was used. ${ }^{(67)}$ This platform consists of three channels. Endothelial cells are seeded in the central channel and tumor cells are seeded on the side channels. The side channels are connected to the central channel by pathways filled with gelatin. After culturing, the cancer cells accumulate in the nearest 


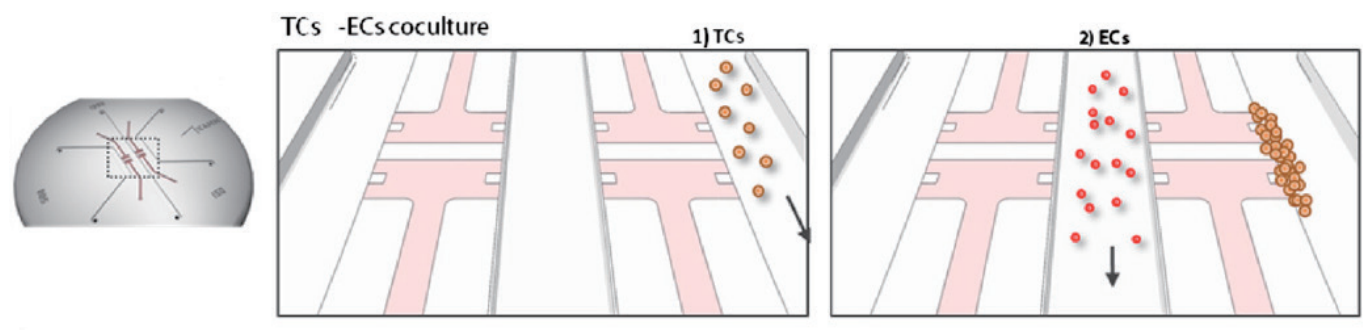

Fig. 8. (Color online) Schematic of tumor-vessel coculture devices. ${ }^{(67)}$

pathway to the vessels, allowing study of transportation and invasion processes. The disease-on-a-chip platform has proven to be an excellent in vitro tool to examine how cancer cells develop in tissues. ${ }^{(76)}$

\subsection{Other organs-on-a-chip}

In addition to major organs, such as the heart, lungs, and liver, kidney ${ }^{(32)}$, splenon ${ }^{(77,78)}$ - and breast ${ }^{(79)}$-on-a-chip devices have been developed. Figure 9 shows the schematics of these three devices. Kidney-on-a-chip devices include a porous membrane where kidney and epithelial cells are cultured on each side. This membrane, which is similar to that used in the lung-on-a-chip device, consists of a central channel and two subchannels - an apical "luminal" channel and a basal "interstitial" space. Compared with traditional microfluidic systems, the exposure of the epithelial cell layer to the shear stress generated by inflow mimics in vivo kidney tubules, promoting epithelial cell polarization and primary cilia formation. This platform is useful for the evaluation of kidney toxicity during drug development.

Breast-on-a-chip and splenon-on-a-chip systems have also utilized microfluidic technology. However, their capacities are limited. The breast-on-a-chip devices only mimic the distribution of anatomical vessels of a breast rather than the whole microenvironment containing glandular, fatty, and vascular tissues. The device can be integrated with other tumor-on-a-chip devices to further mimic tumor development in the breast. The splenon-on-a-chip system only simulates the blood filtering function of the in vivo splenon using a microfluidic two-phase flow structure. Although it does not precisely mimic the corresponding tissues at a microenvironmental level, such devices simulate anatomical geometries or biological functions using microfluidic techniques. The high-level similarities between organ-on-a-chip devices and genuine organs are helpful for researchers who seek to develop a human-on-a-chip system - a comprehensive in vitro system to study drug effects on the whole human body.

\section{Challenges and Future Direction}

Despite the realization of many organ-on-a-chip systems to functionally mimic many tissues and organs in recent years, much work remains ahead to manufacture organ-on-a-chip systems. In general, the ultimate goal of an organ-on-a-chip system 


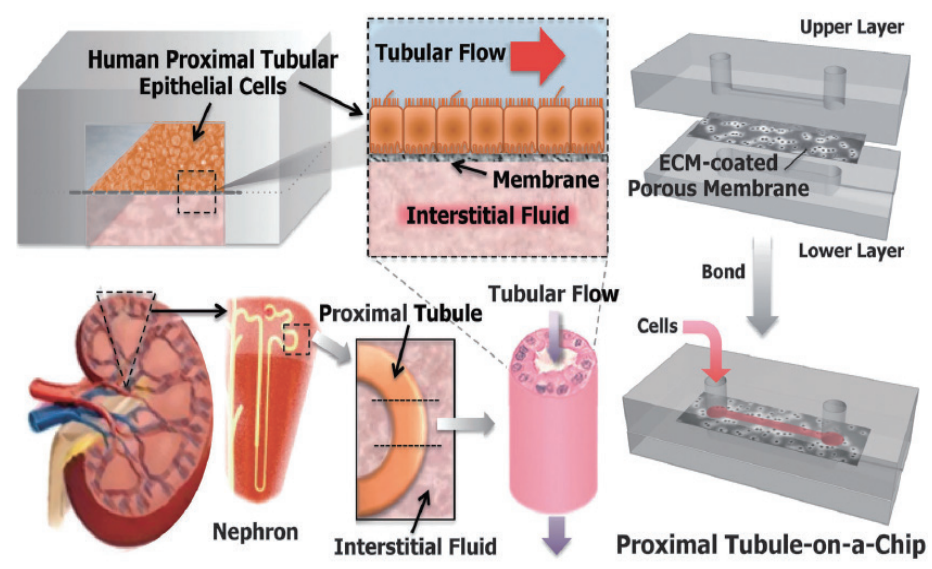

(a)

(b)

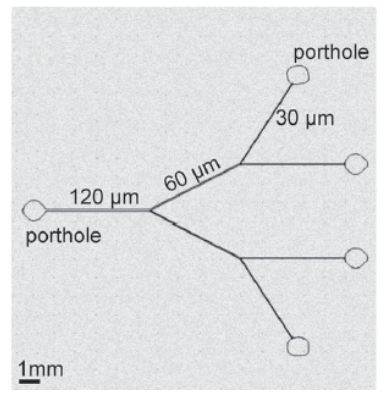

(c)

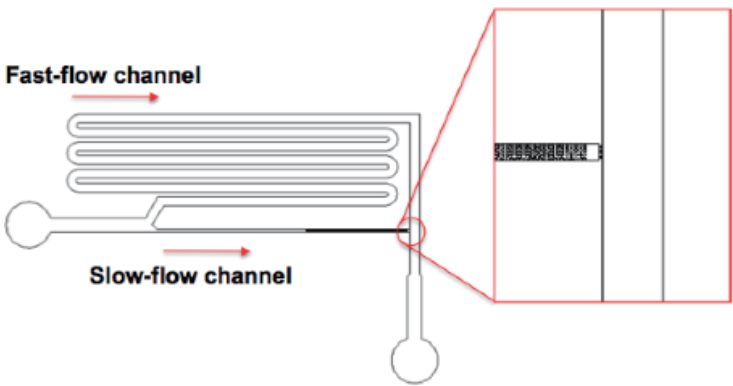

(d)

Fig. 9. (Color online) Kidney-, splenon-, and breast-on-a-chip systems. (a) Conceptual design of kidney tubule-on-a-chip. ${ }^{(32)}$ (b) Fabrication of kidney-on-a-chip. ${ }^{(32)}$ (c) Schematic of breast-on-achip. ${ }^{(79)}$ (d) Layout of splenon-on-a-chip. ${ }^{(78)}$

is to build human-on-a-chip systems using human cells and tissues that are capable of replacing animal testing. To achieve this goal, the feasibility, reliability, controllability, and observability of organ-on-a-chip systems must be improved, to make them comprehensive platforms for toxicological and metabolic tests of drugs.

In terms of feasibility, the verification of the design, mathematical and physical models with acceptable accuracy have to be investigated. Although CFD have been developed for many years and there are many CFD-based simulation models, ${ }^{(75)}$ the analysis of flows for living cells in composite cell media remains challenging. This problem is also related to the mathematical analysis of cell properties. Current models focus on a single property of cells or cell behaviors, such as fusion, ${ }^{(80-82)}$ ECM-cell 
interactions, ${ }^{(83)}$ and cell-cell interactions. ${ }^{(84)}$ Those simple models require further development to simulate in vivo cell activities precisely. Artificial neural network and machine learning technology can be applied to address the demand of complex models of human-on-a-chip systems. Such algorithms are also able to aid and optimize the design of human-on-a-chips. Taken together, to develop better organ-on-a-chip devices, researchers need to understand the biophysical and biochemical properties of organs, and express the nature of organs using mathematical forms. Moreover, these expressions should be modeled by computers using either of the numerical computation methods (Monte Carlo and finite element methods, FEM) or abstract data mining algorithm to verify and accelerate the detailed design process.

For reliability, the long-term stability of organ-on-a-chip systems needs to be verified. It might not become a potential inducer of cell canceration or lead to any undesired change in cell properties and gene integrity. Also, these systems are required to maintain structural integrity after long-term exposure to chemical and biological solutions and materials. Widely used PDMS materials need to be improved or replaced to achieve higher resistance to chemicals, as well as maintain high compatibility with soft lithography techniques and biological cells. ${ }^{(85)}$

In terms of controllability, the automation of both organ-on-a-chip fabrication and screening need to be standardized and optimized. Bio-computer-aided design and manufacturing (Bio-CAD and Bio-CAM) should be introduced to accelerate the design flow of organ-on-a-chip systems. In addition, to simulate drug metabolism, novel interconnection methods to integrate several organ-on-a-chip devices as well as the methods to better mimic an organ's inherent functions at different levels are highly desired. The future designs of the human-on-a-chip systems will require the adoption of modularized methodology — a method to rapidly build highly reliable systems with a variety of desired functions. A practical system will be realized by combining various prebuilt modules. This modularized technique requires researchers developing organ-ona-chip systems to provide well-designed libraries covering all levels and types of organs.

For observability, real-time highly efficient monitoring will remain a challenge for a long time. An ideal human-on-a-chip platform needs to include many basic sensors to record microenvironmental signals, such as $\mathrm{pH}$ and ion concentration. Customized sensors for detecting proteins, chemicals, and even circulating tumor cells are also required. It is difficult to develop sensors at the microscale with high accuracy, biocompatibility, and compatibility using a soft lithography process. Biosensors are potential solutions for this problem. ${ }^{(8)}$ However, both the sensitivity and throughput rate of biosensors need to be improved.

In the long term, organ-on-a-chip systems could be more than an alternative solution for drug screening and delivery test. This approach also has the potential to be used as a high-speed, highly customized, and noninvasive platform to address patient-topatient variation in drug response. In addition to clinical applications, organ-on-achip systems can also play important roles in biological research. Combined with other nanofabrication technologies, an organ-on-a-chip platform can be comprehensive, highly synthesized platform for cell-, tissue-, and organ-level screening and characterization. This platform will help biologists understand the mystery of microenvironments inside human beings, animals, and plants. 
In conclusion, organ-on-a-chip platforms are in vitro microfabricated devices mimicking organ functions. These platforms may be promising tools for discovering drugs and probing cellular functions at the microscale for cellular and molecular biology research.

\section{Acknowledgements}

This work was supported by 2013 Research Funds of Hyundai Heavy Industries for University of Ulsan.

\section{References}

1 C. Moraes, G. Mehta, S. C. Lesher-Perez and S. Takayama: Ann. Biomed. Eng. 40 (2012) 1211.

2 L. Zimmerlin, A. D. Donnenberg, J. P. Rubin, P. Basse, R. J. Landreneau and V. S. Donnenberg: Tissue Eng. Part A 17 (2011) 93.

3 S. Wachsmann-Hogiu, T. Weeks and T. Huser: Curr. Opin. Biotechnol. 20 (2009) 63.

4 S. Lee, J. M. Greve, D. Leaffer, L. Lollini, P. Bailey, G. E. Gold and S. Biswal: NMR Biomed. 21 (2008) 527.

5 H. HogenEsch and A. Y. Nikitin: J. Controlled Release 164 (2012) 183.

6 C. Lo Celso, J. W. Wu and C. P. Lin: J. Biophotonics 2 (2009) 619.

7 D. L. Stirland, J. W. Nichols, S. Miura and Y. H. Bae: J. Controlled Release 172 (2013) 1045.

8 N. S. Bhise, J. Ribas, V. Manoharan, Y. S. Zhang, A. Polini, S. Massa, M. R. Dokmeci and A. Khademhosseini: J. Controlled Release 190 (2014) 82.

9 N. S. Bhise, R. S. Gray, J. C. Sunshine, S. Htet, A. J. Ewald and J. J. Green: Biomaterials 31 (2010) 8088.

10 D. C. Duffy, J. C. McDonald, O. J. Schueller and G. M. Whitesides: Anal. Chem. 70 (1998) 4974.

11 E. Leclerc, K. S. Furukawa, F. Miyata, Y. Sakai, T. Ushida and T. Fujii: Biomaterials 25 (2004) 4683.

12 D. Huh, Y. Torisawa, G. A Hamilton, H. J. Kim and D. E. Ingber: Lab Chip 12 (2012) 2156.

13 A. M. Ghaemmaghami, M. J. Hancock, H. Harrington, H. Kaji and A. Khademhosseini: Drug Discovery Today 17 (2012) 173.

14 T. Ross: Physican Executive 31 (2005) 48.

15 B. Djulbegovic, H. Outcome and H. L. Mofitt: J. Am. Med. Assoc. 311 (2014) 355.

16 N. S. Bhise, R. B. Shmueli, J. C. Sunshine, S. Y. Tzeng and J. J. Green: Expert Opin. Drug Delivery 8 (2011) 485.

17 P. Zorlutuna, N. E. Vrana and A. Khademhosseini: IEEE Rev. Biomed. Eng. 6 (2013) 47.

18 B. J. Papenburg, J. Liu, G. A. Higuera, A. M. C. Barradas, J. de Boer, C. A. van Blitterswijk, M. Wessling and D. Stamatialis: Biomaterials 30 (2009) 6228.

19 Y.-C. Tung, A. Y. Hsiao, S. G. Allen, Y. Torisawa, M. Ho and S. Takayama: Analyst 136 (2011) 473.

20 S. Wilkening, F. Stahl and A. Bader: Drug Metab. Dispos. 31 (2003) 1035.

21 J. M. Karp, J. Yeh, G. Eng, J. Fukuda, J. Blumling, K.-Y. Suh, J. Cheng, A. Mahdavi, J. Borenstein, R. Langer and A. Khademhosseini: Lab Chip 7 (2007) 786.

22 N. G. Patel and G. Zhang: Organogenesis 10 (2014) 170.

23 N. J. Douville, P. Zamankhan, Y.-C. Tung, R. Li, B. L. Vaughan, C.-F. Tai, J. White, P. J. Christensen, J. B. Grotberg and S. Takayama: Lab Chip 11 (2011) 609. 
24 D. Huh, B. D. Matthews, A. Mammoto, M. Montoya-Zavala, H. Y. Hsin and D. E. Ingber: Science 328 (2010) 1662.

25 S. K. Mahto, T. H. Yoon and S. W. Rhee: Biomicrofluidics 4 (2010) 1.

26 E. C. Cho, Q. Zhang and Y. Xia: Nat. Nanotechnol. 6 (2011) 385.

27 T. Lammers, F. Kiessling, W. E. Hennink and G. Storm: J. Controlled Release 161 (2012) 175 .

28 U.S. Food and Drug Administration: Guidance for Industry Safety Testing of Drug Metabolites (2008) pp. 1-11.

29 T. S. Fröde and Y. S. Medeiros: J. Ethnopharmacol. 115 (2008) 173.

30 B. Kwak, A. Ozcelikkale, C. S. Shin, K. Park and B. Han: J. Controlled Release 194 (2014) 157.

31 P. J. Lee, P. J. Hung and L. P. Lee: Biotechnol. Bioeng. 97 (2007) 1340.

32 K.-J. Jang, A. P. Mehr, G. A. Hamilton, L. A. McPartlin, S. Chung, K.-Y. Suh and D. E. Ingber: Integr. Biol. 5 (2013) 1119.

33 D. Huh, G. A. Hamilton and D. E. Ingber: Trends Cell Biol. 21 (2011) 745.

34 B. Jiang, W. Zheng, W. Zhang and X. Jiang: Sci. China Chem. 57 (2013) 356.

35 S. N. Bhatia and D. E. Ingber: Nat. Biotechnol. 32 (2014) 760.

36 A. Bernard, J. P. Renault, B. Michel, H. R. Bosshard and E. Delamarche: Adv. Mater. 12 (2000) 1067.

37 H. V. Prentice-Mott, C.-H. Chang, L. Mahadevan, T. J. Mitchison, D. Irimia and J. V. Shah: Proc. Natl. Acad. Sci. U.S.A. 110 (2013) 21006.

38 M. Radisic, W. Deen, R. Langer and G. Vunjak-Novakovic: Am. J. Physiol. Heart Circ. Physiol. 288 (2005) H1278.

39 E. Cimetta, C. Cannizzaro, R. James, T. Biechele, R. T. Moon, N. Elvassore and G. VunjakNovakovic: Lab Chip 10 (2010) 3277.

40 J. Kusunose, H. Zhang, M. K. J. Gagnon, T. Pan, S. I. Simon and K. W. Ferrara: Ann. Biomed. Eng. 41 (2013) 89.

41 N. Korin, M. Kanapathipillai, B. D. Matthews, M. Crescente, A. Brill, T. Mammoto, K. Ghosh, S. Jurek, S. A. Bencherif, D. Bhatta, A. U. Coskun, C. L. Feldman, D. D. Wagner and D. E. Ingber: Science 337 (2012) 738.

42 D. Kim, S. Finkenstaedt-Quinn, K. R. Hurley, J. T. Buchman and C. L. Haynes: Analyst 139 (2014) 906.

43 J. M. Rosano, N. Tousi, R. C. Scott, B. Krynska, V. Rizzo, B. Prabhakarpandian, K. Pant, S. Sundaram and M. F. Kiani: Biomed. Microdevices 11 (2009) 1051.

44 K. Namdee, A. J. Thompson, P. Charoenphol and O. Eniola-adefeso: Langmuir 29 (2013) 2530.

45 S. P. Samuel, N. Jain, F. O. Dowd, T. Paul, V. A. Gerard, Y. K. Gun and A. Prina-Mello: Int. J. Nanomedicine 7 (2012) 2943.

46 G. Lamberti, Y. Tang, B. Prabhakarpandian, Y. Wang, K. Pant, M. F. Kiani and B. Wang: Microvasc. Res. 89 (2013) 107.

47 B. Tesfamariam and A. F. DeFelice: Vasc. Pharmacol. 46 (2007) 229.

48 Y.-S. J. Li, J. H. Haga and S. Chien: J. Biomech. 38 (2005) 1949.

49 S. Srigunapalan, C. Lam, A. R. Wheeler and C. A. Simmons: Biomicrofluidics 5 (2011) 13409.

50 W. Zheng, B. Jiang, D. Wang, W. Zhang, Z. Wang and X. Jiang: Lab Chip 12 (2012) 3441.

51 K. Ohashi, T. Yokoyama, M. Yamato, H. Kuge, H. Kanehiro, M. Tsutsumi, T. Amanuma, H. Iwata, J. Yang, T. Okano and Y. Nakajima: Nat. Med. 13 (2007) 880.

52 S.-A. Lee, D. Y. No, E. Kang, J. Ju, D.-S. Kim and S.-H. Lee: Lab Chip 13 (2013) 3529.

53 K. Domansky, W. Inman, J. Serdy, A. Dash, M. H. M. Lim and L. G. Griffith: Lab Chip 10 (2010) 51 . 
54 Y.-C. Toh, T. C. Lim, D. Tai, G. Xiao, D. van Noort and H. Yu: Lab Chip 9 (2009) 2026.

55 Y.-C. Toh, C. Zhang, J. Zhang, Y. M. Khong, S. Chang, V. D. Samper, D. van Noort, D. W. Hutmacher and H. Yu: Lab Chip 7 (2007) 302.

56 V. N. Goral, Y.-C. Hsieh, O. N. Petzold, J. S. Clark, P. K. Yuen and R. A. Faris: Lab Chip 10 (2010) 3380 .

57 S. R. Shin, S. M. Jung, M. Zalabany, K. Kim, P. Zorlutuna, S. Kim, M. Nikkhah, M. Khabiry, M. Azize, J. Kong, K. Wan and T. Palacios: ACS Nano 7 (2013) 2369.

58 A. Grosberg, P. W. Alford, M. L. McCain and K. K. Parker: Lab Chip 11 (2011) 4165.

59 A. A. Werdich, E. A. Lima, B. Ivanov, I. Ges, M. E. Anderson, J. P. Wikswo and F. J. Baudenbacher: Lab Chip 4 (2004) 357.

60 W. Cheng, N. Klauke, H. Sedgwick, G. L. Smith and J. M. Cooper: Lab Chip 6 (2006) 1424.

61 L. Zhao, Z. Wang, S. Fan, Q. Meng, B. Li, S. Shao and Q. Wang: Biomed. Microdevices 12 (2010) 325 .

62 C.-W. Huang, J.-Y. Cheng, M.-H. Yen and T.-H. Young: Biosens. Bioelectron. 24 (2009) 3510.

63 Z. Xu, Y. Gao, Y. Hao, E. Li, Y. Wang, J. Zhang, W. Wang, Z. Gao and Q. Wang: Biomaterials 34 (2013) 4109.

64 H.-S. Hou, H.-F. Tsai, H.-T. Chiu and J.-Y. Cheng: Biomicrofluidics 8 (2014) 052007.

65 T. Huang, C.-P. Jia, Jun-Yang, W.-J. Sun, W.-T. Wang, H.-L. Zhang, H. Cong, F.-X. Jing, H.-J. Mao, Q.-H. Jin, Z. Zhang, Y.-J. Chen, G. Li, G.-X. Mao and J.-L. Zhao: Biosens. Bioelectron. 51 (2014) 213.

66 C. Long, C. Finch, M. Esch, W. Anderson, M. Shuler and J. Hickman: Ann. Biomed. Eng. 40 (2012) 1255.

67 I. K. Zervantonakis, C. R. Kothapalli, S. Chung, R. Sudo and R. D. Kamm: Biomicrofluidics 5 (2011) 13406.

68 K.-A. Hyun, T. Y. Lee and H.-I. Jung: Anal. Chem. 85 (2013) 4439.

69 P. Li, Z. S. Stratton, M. Dao, J. Ritz and T. J. Huang: Lab Chip 13 (2013) 602.

70 S. Yamamura, S. Yatsushiro, K. Abe, Y. Baba and M. Kataoka: J. Phys. Conf. Ser. 352 (2012) 012041.

71 C. Kim, J. H. Bang, Y. E. Kim, S. H. Lee and J. Y. Kang: Lab Chip 12 (2012) 4135.

72 D. Sun, J. Lu, Z. Chen, Y. Yu and Y. Li: Microfluid. Nanofluidics 17 (2014) 831.

73 M. Alshareef, N. Metrakos, E. Juarez Perez, F. Azer, F. Yang, X. Yang and G. Wang: Biomicrofluidics 7 (2013) 11803.

74 N. T. Elliott and F. Yuan: Biotechnol. Bioeng. 109 (2012) 1326.

75 J. Tan, S. Shah, A. Thomas, H. D. Ou-Yang and Y. Liu: Microfluid. Nanofluidics 14 (2013) 77.

76 P. A. Vidi, T. Maleki, M. Ochoa, L. Wang, S. M. Clark, J. F. Leary and S. A. Lelièvre: Lab Chip 14 (2014) 172.

77 M. Bernabeu, A. Elizalde and M. De Niz: XIII Mediterranean Conference on Medical and Biological Engineering and Computing 2013, eds. R. Roa and M. Laura (Springer International Publishing, Seville, 2014) pp. 884-887.

78 L. G. Rigat-Brugarolas, A. Elizalde-Torrent, M. Bernabeu, M. De Niz, L. Martin-Jaular, C. Fernandez-Becerra, A. Homs-Corbera and J. Samitier: Lab Chip 14 (2014) 1715.

79 M. M. G. Grafton, L. Wang, P.-A. Vidi, J. Leary and S. A. Lelièvre: Integr. Biol. 3 (2011) 451.

80 X. Yang, V. Mironov and Q. Wang: J. Theor. Biol. 303 (2012) 110.

81 X. Yang, Y. Sun and Q. Wang: J. Biomech. Eng. 135 (2013) 71005.

82 G. L. Thomas, V. Mironov, A. Nagy-Mehez and J. C. M. Mombach: Phys. A Stat. Mech. Appl. 395 (2014) 247. 
83 C. S. Ki, T.-Y. Lin, M. Korc and C.-C. Lin: Biomaterials 35 (2014) 9668.

84 T. Abdulla, R. A. Imms, J.-M. Schleich and R. Summers: Proc. Annu. Int. Conf. IEEE Eng. Med. Biol. Soc. 2011 (IEEE, Boston, 2011) p. 449.

85 E. Berthier, E. W. K. Young and D. Beebe: Lab Chip 12 (2012) 1224.

86 A. Khademhosseini, R. Langer, J. Borenstein and J. P. Vacanti: Proc. Natl. Acad. Sci. U.S.A. 103 (2006) 2480.

87 H. Tekin, M. Anaya, M. D. Brigham, C. Nauman, R. Langer and A. Khademhosseini: Lab Chip 10 (2010) 2411.

88 D. Therriault, S. R. White and J. A Lewis: Nat. Mater. 2 (2003) 265. 